\title{
Reproducibility of Repeated Breathhold and Impact of Breathhold Failure in Whole Breast and Regional Nodal Irradiation in Prone Crawl Position
}

Pieter Deseyne ( $\nabla$ pieter.deseyne@uzgent.be )

Ghent University Hospital

Bruno Speleers

Ghent University

Leen Paelinck

Ghent University Hospital

Werner De Gersem

Ghent University Hospital

Wilfried De Neve

Ghent University

Max Schoepen

Ghent University

Annick Van Greveling

Ghent University Hospital

Hans Van Hulle

Ghent University

Vincent Vakaet

Ghent University

Giselle Post

Ghent University

Chris Monten

Ghent University Hospital

Herman Depypere

Ghent University Hospital

Liv Veldeman

Ghent University Hospital

Research Article

Keywords: Reproducibility, breathhold, OAR, FTBH 
Posted Date: March 1st, 2021

DOl: https://doi.org/10.21203/rs.3.rs-199471/v1

License: (c) (i) This work is licensed under a Creative Commons Attribution 4.0 International License. Read Full License

Version of Record: A version of this preprint was published at Scientific Reports on February 3rd, 2022. See the published version at https://doi.org/10.1038/s41598-022-05957-7. 


\section{Abstract}

In whole breast and regional nodal irradiation $(\mathrm{WB}+\mathrm{RNI})$, breathhold increases organ at risk (OAR) sparing. $\mathrm{WB}+\mathrm{RNI}$ is usually performed in supine position, because positioning materials obstruct beam paths in prone position. Recent advancements allow prone WB + RNI (pWB + RNI) with increased sparing of OARs compared to supine WB + RNI. We evaluate positional and dosimetrical impact of repeated breathhold (RBH) and failure to breathhold (FTBH) in pWB + RNI.

Twenty left-sided breast cancer patients were scanned twice in breathhold (baseline and RBH) and once free breathing (i.e. FTBH). Positional impact was evaluated using overlap index (OI) and Dice similarity coefficient (DSC). Dosimetrical impact was assessed by beam transposition from the baseline plan.

Mean OI and DSC ranges were $0.01-0.98$ and $0.01-0.92$ for $\mathrm{FTBH}$, and $0.73-1$ and $0.69-1$ for RBH. Dosimetric impact of RBH was negligible. FTBH significantly decreased minimal dose to CTV WBI, level II and the internal mammary nodes, with adequate mean doses. FTBH significantly increased heart, LAD, left lung and esophagus dose.

Ol and DSC for RBH and FTBH show reproducible large ROI positions. Small ROls show poor overlap. FTBH maintained adequate target coverage but increased heart, $L A D$, ipsilateral lung and esophagus dose. $\mathrm{RBH}$ is a robust technique in pWB $+\mathrm{RNI}$.

\section{Introduction}

Therapeutic evolution has led to an increase in long-term survival for breast cancer patients. As survival increases, so does the impact of treatment-related side effects. The effects of radiotherapy on the occurrence of major cardiac events have been described ${ }^{1}$. For this reason, several techniques have been proposed to limit the dose to the heart. The most important are breathhold ${ }^{2,3}$ and prone positioning ${ }^{4,5}$, two techniques that can be combined in whole breast irradiation (WBI).

Breathhold can be used in whole breast and regional nodal irradiation (WB $+\mathrm{RNI})$ to improve sparing of normal tissues ${ }^{6,7}$, but because the breast board on which the patient is positioned obstructs beam access to the regional nodal areas, the prone position is almost exclusively used in WBI, and not in WB + RNI. However, recent trials have shown that $\mathrm{WB}+\mathrm{RNI}$ in prone position is not only possible but comes with the same benefits of prone positioning observed in $\mathrm{WBI}{ }^{8-12}$. This opens perspectives to once again combine breathhold with prone positioning. Contrary to the breast in prone position ${ }^{13}$, nodal target volume locations may vary significantly between shallow breathing and breathhold, requiring strict breathhold monitoring to avoid missing the target. These observations have been reported for supine position 14,15 , but such data are non-existent for prone positioning. This trial investigates the reproducibility of breathhold and the impact of the observed changes on dose coverage. The variation in position of organs at risk (OARs) and target volumes (TVs) between breathhold and free breathing in 
prone position is evaluated, to estimate the impact of patients that do not succeed in maintaining breathhold during treatment.

\section{Materials And Method}

\section{Study design}

This study investigates the feasibility and intra-fraction reproducibility of the breathhold technique in prone position for patients requiring WB + RNI. We included 20 left-sided breast cancer patients referred to our center for WBI and used their simulation imaging to plan WBI + RNI treatment. In addition to a free breathing computed tomography (CT) scan, patients underwent 2 voluntary deep inspiration breathhold $\mathrm{BH})$ scans at simulation. The second $\mathrm{BH}$ scan was performed to evaluate reproducibility of the procedure, and had no therapeutic implications. The trial was approved by the Ghent University Hospital Ethics Board (reference number: EC-UZ-2016/0351, Belgian Registration Number: B670201628048). All patients in our research voluntarily joined the study and informed consent was obtained from all participants before inclusion. The research was performed in accordance with relevant guidelines and regulations.

\section{Prone crawl position}

Patients were positioned in prone position on the crawl couch, which was specifically designed for WB + RNI. The device is described elsewhere and yields lower doses to OARs while maintaining target coverage when compared to supine $\mathrm{WB}+\mathrm{RNI}{ }^{9,12,16}$.

\section{Simulation and breathhold}

All patients underwent CT simulation with a unilateral bra [Tricolast, Deinze, Belgium] that retracts the contralateral breast from the TVs. We used a slice thickness of $5 \mathrm{~mm}$ for image acquisition. Each patient underwent a free breathing CT scan and a BH scan, as well as an additional low dose repeated breathhold (RBH) scan. Patient position nor scan range were altered between each scan, assuring that the DICOM coordinate system, indicated by the frame of reference unique identifier of the different scans, remained identical. The RBH manoeuvre was monitored using 2 Respisens magnetic sensors (Nomics, Angleur, Belgium) placed on the breast couch and thoracic wall ${ }^{17}$. No IV-contrast was administered. This provided us with a set of 3 scans of distinct clinical situations for each patient, namely $\mathrm{BH}, \mathrm{RBH}$ and failure to breathhold (FTBH). We will further refer to these names when describing these clinical situations, where we consider $\mathrm{BH}$ to be the index situation and $\mathrm{RBH}$ and $\mathrm{FTBH}$ the comparative situations.

\section{Treatment design and prescribed dose}

The prescription dose was 40.05 Gy in 15 fractions to the whole breast, axillary levels II-III-IV and internal mammary nodes. Level I was excluded from the target volumes because this level is cleared during axillary lymph node dissection. 


\section{Delineation}

An extrapolation of the accepted guidelines for delineation of the breast and lymphatic regions from the contouring guidelines as proposed by the ESTRO and PROCAB groups ${ }^{18-21}$ was used as there are no generally accepted guidelines for delineation in prone position. The axillary levels I-II-III (LNN I, LNN II and LNN III), the supraclavicular fossa (LNN IV) and internal mammary nodes (LNN MI) were delineated separately, resulting in 5 separate nodal regions. The heart and left anterior descending coronary artery (LAD) were delineated in accordance with guidelines proposed by Feng et al. ${ }^{22}$. The contralateral breast was delineated up to the skin. Where OARs were not visible on certain CT-slices, interpolation was used. Because all images were acquired within the same session without repositioning, co-registration by DICOM coordinates was performed. Delineations were first performed on the FTBH scan, and were copied to the $\mathrm{BH}$ scan and adapted where necessary. This process was repeated between $\mathrm{BH}$ and the $\mathrm{RBH}$ scan.

\section{Overlap indicators}

After contouring the TVs and OARs, we performed a paired comparison of the total volumes of the breast, individual nodal regions, heart, $\mathrm{LAD}$, and contralateral breast between $\mathrm{BH}$ and $\mathrm{RBH}$ scans, and between FTBH and BH scans. We assessed the spatial overlap between contoured volumes on different scans using the Dice similarity coefficient (DSC) ${ }^{23}$ and the overlap index $(\mathrm{OI})^{13}$. Both have been used to describe reproducibility and accuracy of delineation.

The DSC is defined as follows:

$D S C=\frac{2 \times(A \cap B)}{A+B}$

whereas the OI is defined as:

$O I=\frac{A \cap B}{A}$

In both formulas, $A$ is the volume of a contoured region on one scan, and B is the volume of the same contoured region on another scan.

These numerical values are a surrogate of the clinical relevance of reproducibility. Therefore, we also investigated dosimetry on these scans to assess the impact of the observed overlap indicators.

\section{Dosimetric estimation of clinical relevance of intra-fraction motion}

A treatment plan was made for the $\mathrm{BH}$ scan for each patient using a previously described technique ${ }^{9}$. The treatment beams for this initial plan were transposed by copying the machine instruction file to the FTBH scan. The dose was then recalculated without re-optimization on the FTBH scan to assess the effect of FTBH. The same was done on the RBH scan to assess the reproducibility of RBH. A separately measured electron-density table was used for dose calculation on the low-dose CT.

\section{Data analysis}


Data was analysed using R version 3.4.1, using two-sided paired significance testing with an a-level of 0.05 .

\section{Results}

\section{Absolute volumes}

Paired T-tests showed a significant absolute volume difference for the contralateral breast, heart, lungs, level I and level II $(\mathrm{P}<0.05)$ between $\mathrm{BH}$ and FTBH scans. Only left and right lung volume was significantly different between $\mathrm{BH}$ and $\mathrm{RBH}$ scans. Table 1 shows the mean volumes and standard deviation for each of the delineated regions of interest (ROIs) on the different scans.

Table 1

Mean volume \pm standard deviation in milliliter for each delineated volume on all scans. $\mathrm{FTBH}=$ scan in failure to breathhold setting, $\mathrm{BH}=$ initial voluntary deep inspiration breathhold scan, $\mathrm{RBH}=$ low dose repeated breathhold scan, $\mathrm{LAD}=$ left anterior descendant coronary artery. ${ }^{*}=\mathrm{P}<0.05$ compared to volume on $\mathrm{BH}$. Contralat. $=$ contralateral.

\begin{tabular}{|llll|}
\hline & FTBH & BH & RBH \\
\hline CTV WBI & $455.62 \pm 298.56$ & $484.59 \pm 347.02$ & $456.74 \pm 298.29$ \\
\hline LNN I & $117.96 \pm 56.88 *$ & $119.23 \pm 57.08$ & $119.68 \pm 57.34$ \\
\hline LNN II & $18.02 \pm 7.74 *$ & $16.22 \pm 7.25$ & $15.90 \pm 7.36$ \\
\hline LNN III & $13.73 \pm 4.19$ & $14.02 \pm 4.64$ & $14.05 \pm 4.62$ \\
\hline LNN IV & $13.11 \pm 2.63$ & $12.35 \pm 3.20$ & $12.41 \pm 3.18$ \\
\hline LNN MI & $8.42 \pm 2.32$ & $7.62 \pm 3.00$ & $7.59 \pm 2.65$ \\
\hline Heart & $648.03 \pm 100.18 *$ & $604.68 \pm 73.64$ & $602.29 \pm 76.84$ \\
\hline LAD & $3.80 \pm 1.72$ & $3.98 \pm 1.77$ & $4.02 \pm 1.66$ \\
\hline Left Lung & $1562.78 \pm 365.86 *$ & $2313.95 \pm 473.40$ & $2275.19 \pm 458.07$ * \\
\hline Right Lung & $1875.46 \pm 394.72 *$ & $2634.98 \pm 458.17$ & $2516.19 \pm 472.28$ * \\
\hline Esophagus & $29.94 \pm 12.20$ & $32.16 \pm 14.55$ & $31.11 \pm 12.34$ \\
\hline Thyroid & $16.39 \pm 14.67$ & $17.28 \pm 15.20$ & $16.69 \pm 15.59$ \\
\hline Contralat. & $618.80 \pm 369.03 *$ & $606.14 \pm 359.42$ & $606.286 \pm 359.73$ \\
\hline breast & & & \\
\hline
\end{tabular}

\section{Overlap measurements}

Figure 1 shows a boxplot of OI and DSC for the different ROIs in the different breathing settings. Regarding OARs, LAD, esophagus and thyroid showed more variability in OI and DSC than the other 
OARs. For TVs, OI and DSC varied more prominently. All ROls with smaller volumes show higher overlap discrepancies. OI and DSC show a high correlation (Pearson's $r=0.98, R^{2}=0.95$ ).

\section{Beam transposition}

DVH parameters for TVs are reported in Table 2 and for OARs in Table 3. Figure 2 and Fig. 3 show mean DVH curves for TVs and OARs, respectively. The TVs showed no significant dosimetric differences for $\mathrm{RBH}$. In FTBH, there were numerically significant but clinically less relevant dosimetric differences for CTV WBI, LNN III and LNN IV. Larger differences were apparent in D95 and D98 of LNN II and LNN MI, which were also significantly reduced in FTBH (Table 2). We report dose to the LNN I region, but did not include this region as a TV. 
Table 2

Target volume dose parameters after transposition of breathhold plan (BH) to repeated breathhold (RBH) and failure to breathhold (FTBH) CT anatomy. We show dose \pm standard deviation. CTV LNN is what we consider the unoperated axilla, i.e. LNN II + LNN III + LNN IV but excluding CTV MI (a TV with separate planning optimization). (LNN I): This region is not included as a target volume in our trial, but we report dose to this region for the interested reader. Dxx: Minimum dose received by $x x \%$ of ROI

volume. NS: not significant

\begin{tabular}{|c|c|c|c|c|c|c|}
\hline ROI & Param & $\mathrm{BH}$ & FTBH & P-value FTBH & RBH & P-value RBH \\
\hline \multirow[t]{5}{*}{ CTV WBI } & Dmean & $40.42 \pm 0.17$ & $40.33 \pm 0.21$ & $P=0.007$ & $40.47 \pm 0.18$ & NS \\
\hline & D02 & $42.59 \pm 0.71$ & $42.44 \pm 0.74$ & $P=0.025$ & $42.63 \pm 0.75$ & NS \\
\hline & D50 & $40.42 \pm 0.13$ & $40.37 \pm 0.18$ & NS & $40.45 \pm 0.16$ & NS \\
\hline & D95 & $38.76 \pm 0.44$ & $38.55 \pm 0.44$ & $P<0.001$ & $38.66 \pm 0.49$ & $P=0.033$ \\
\hline & D98 & $38.00 \pm 0.66$ & $37.69 \pm 0.70$ & $P=0.005$ & $37.90 \pm 0.75$ & NS \\
\hline \multirow[t]{5}{*}{ LNN II } & Dmean & $40.40 \pm 0.44$ & $40.26 \pm 0.55$ & NS & $40.42 \pm 0.36$ & NS \\
\hline & D02 & $41.62 \pm 0.85$ & $41.81 \pm 0.73$ & NS & $41.70 \pm 0.84$ & NS \\
\hline & D50 & $40.40 \pm 0.43$ & $40.51 \pm 0.38$ & NS & $40.43 \pm 0.38$ & NS \\
\hline & D95 & $39.45 \pm 0.33$ & $38.06 \pm 2.30$ & $P=0.016$ & $39.35 \pm 0.39$ & NS \\
\hline & D98 & $39.09 \pm 0.48$ & $36.57 \pm 3.36$ & $P=0.003$ & $38.90 \pm 0.61$ & NS \\
\hline \multirow[t]{5}{*}{ LNN III } & Dmean & $40.50 \pm 0.53$ & $40.49 \pm 0.48$ & NS & $40.56 \pm 0.65$ & NS \\
\hline & D02 & $42.18 \pm 0.98$ & $42.24 \pm 1.00$ & NS & $42.38 \pm 1.11$ & $P=0.008$ \\
\hline & D50 & $40.48 \pm 0.52$ & $40.53 \pm 0.45$ & NS & $40.55 \pm 0.61$ & NS \\
\hline & D95 & $39.23 \pm 0.45$ & $38.98 \pm 0.51$ & $P=0.023$ & $39.12 \pm 0.86$ & NS \\
\hline & D98 & $38.66 \pm 0.93$ & $38.34 \pm 0.81$ & NS & $38.41 \pm 1.55$ & NS \\
\hline \multirow[t]{5}{*}{ LNN IV } & Dmean & $40.44 \pm 0.49$ & $40.46 \pm 0.96$ & NS & $40.46 \pm 0.68$ & NS \\
\hline & D02 & $42.48 \pm 0.98$ & $43.01 \pm 1.23$ & $P=0.027$ & $42.64 \pm 1.25$ & NS \\
\hline & D50 & $40.48 \pm 0.48$ & $40.63 \pm 0.69$ & NS & $40.51 \pm 0.69$ & NS \\
\hline & D95 & $38.67 \pm 1.16$ & $37.77 \pm 2.50$ & NS & $38.60 \pm 1.17$ & NS \\
\hline & D98 & $37.51 \pm 2.98$ & $36.34 \pm 3.50$ & NS & $37.54 \pm 2.65$ & NS \\
\hline \multirow[t]{4}{*}{ LNN MI } & Dmean & $40.67 \pm 0.36$ & $40.23 \pm 1.23$ & NS & $40.46 \pm 0.76$ & NS \\
\hline & D02 & $42.62 \pm 0.82$ & $43.37 \pm 1.75$ & NS & $42.58 \pm 1.05$ & NS \\
\hline & D50 & $40.65 \pm 0.38$ & $40.55 \pm 1.07$ & NS & $40.49 \pm 0.76$ & NS \\
\hline & D95 & $39.14 \pm 0.22$ & $36.09 \pm 4.25$ & $P=0.007$ & $38.69 \pm 1.12$ & NS \\
\hline
\end{tabular}




\begin{tabular}{|c|c|c|c|c|c|c|}
\hline ROI & Param & $\mathrm{BH}$ & FTBH & P-value FTBH & $\mathrm{RBH}$ & P-value RBH \\
\hline & D98 & $38.45 \pm 0.47$ & $33.90 \pm 5.57$ & $P=0.003$ & $37.93 \pm 1.63$ & NS \\
\hline \multirow[t]{5}{*}{ CTV LNN } & Dmean & $40.44 \pm 0.33$ & $40.36 \pm 0.47$ & NS & $40.47 \pm 0.42$ & NS \\
\hline & D02 & $42.33 \pm 0.91$ & $42.65 \pm 0.96$ & NS & $42.48 \pm 1.04$ & NS \\
\hline & D50 & $40.40 \pm 0.27$ & $40.50 \pm 0.35$ & NS & $40.46 \pm 0.35$ & NS \\
\hline & D95 & $39.16 \pm 0.26$ & $38.03 \pm 1.69$ & $P=0.010$ & $38.97 \pm 0.58$ & NS \\
\hline & D98 & $38.45 \pm 0.93$ & $36.44 \pm 2.78$ & $P=0.007$ & $38.27 \pm 1.09$ & NS \\
\hline \multirow[t]{5}{*}{$(\mathrm{LNN} \mathrm{I})$} & Dmean & $20.08 \pm 4.95$ & $20.14 \pm 5.15$ & NS & $20.03 \pm 4.98$ & NS \\
\hline & D02 & $40.66 \pm 0.75$ & $40.92 \pm 0.78$ & $P=0.012$ & $40.74 \pm 0.81$ & NS \\
\hline & D50 & $17.61 \pm 7.93$ & $17.80 \pm 8.12$ & NS & $17.46 \pm 7.93$ & $P=0.021$ \\
\hline & D95 & $3.95 \pm 4.02$ & $3.82 \pm 3.56$ & NS & $3.92 \pm 3.94$ & NS \\
\hline & D98 & $3.08 \pm 3.07$ & $3.06 \pm 2.86$ & NS & $3.07 \pm 2.98$ & NS \\
\hline
\end{tabular}

Regarding OARs, there were no relevant dosimetrical differences for $\mathrm{RBH}$, with the exception of the $\mathrm{V} 30$ to the thyroid. In FTBH, there were significant dose differences for all OARs except for thyroid and right lung (Table 3). 
Table 3

Organ at risk dose parameters after transposition of breathhold plan $(\mathrm{BH})$ to second breathhold $(\mathrm{RBH})$ and failure ot breathhold (FTBH) CT anatomy. We show dose \pm standard deviation. Dxx: Minimum dose received by $x x \%$ of ROI volume, Vxx: volume percentage receiving at least Xx Gy. NS: not significant

\begin{tabular}{|c|c|c|c|c|c|c|}
\hline ROI & & $\mathrm{BH} 1$ & FTBH & $\begin{array}{l}\text { P-value } \\
\text { FTBH }\end{array}$ & RBH & $\begin{array}{l}\text { P-value } \\
\text { RBH }\end{array}$ \\
\hline \multirow[t]{6}{*}{ Heart } & D02 & $\begin{array}{l}14.09 \pm \\
8.93\end{array}$ & $\begin{array}{l}24.46 \pm \\
7.16\end{array}$ & $P<0.001$ & $\begin{array}{l}14.21 \pm \\
8.93\end{array}$ & NS \\
\hline & Dmean & $2.47 \pm 0.97$ & $3.96 \pm 1.35$ & $P<0.001$ & $2.50 \pm 0.98$ & NS \\
\hline & V5 & $7.79 \pm 5.68$ & $\begin{array}{l}16.87 \pm \\
7.68\end{array}$ & $P<0.001$ & $7.97 \pm 5.88$ & NS \\
\hline & V10 & $3.62 \pm 3.61$ & $9.59 \pm 5.59$ & $P<0.001$ & $3.76 \pm 3.62$ & NS \\
\hline & V20 & $1.36 \pm 1.62$ & $3.96 \pm 2.82$ & $P<0.001$ & $1.39 \pm 1.65$ & NS \\
\hline & V30 & $0.45 \pm 0.57$ & $1.44 \pm 1.25$ & $P<0.001$ & $0.45 \pm 0.57$ & NS \\
\hline \multirow[t]{6}{*}{ LAD } & D02 & $\begin{array}{l}14.22 \pm \\
10.16\end{array}$ & $\begin{array}{l}21.22 \pm \\
9.90\end{array}$ & $P<0.001$ & $\begin{array}{l}15.01 \pm \\
9.80\end{array}$ & NS \\
\hline & Dmean & $5.99 \pm 4.19$ & $8.33 \pm 4.08$ & $P=0.004$ & $6.06 \pm 3.94$ & NS \\
\hline & V5 & $\begin{array}{l}31.20 \pm \\
25.57\end{array}$ & $\begin{array}{l}48.45 \pm \\
23.41\end{array}$ & $P<0.001$ & $\begin{array}{l}32.30 \pm \\
24.50\end{array}$ & NS \\
\hline & V10 & $\begin{array}{l}15.45 \pm \\
20.14\end{array}$ & $\begin{array}{l}27.50 \pm \\
19.80\end{array}$ & $P=0.012$ & $\begin{array}{l}16.18 \pm \\
19.69\end{array}$ & NS \\
\hline & V20 & $\begin{array}{l}6.18 \pm \\
14.28\end{array}$ & $\begin{array}{l}10.03 \pm \\
13.76\end{array}$ & NS & $\begin{array}{l}6.44 \pm \\
13.22\end{array}$ & NS \\
\hline & V30 & $\begin{array}{l}1.958 \pm \\
5.30\end{array}$ & $2.52 \pm 4.63$ & NS & $1.42 \pm 4.20$ & NS \\
\hline \multirow[t]{6}{*}{ Left Lung } & D02 & $\begin{array}{l}32.23 \pm \\
2.03\end{array}$ & $\begin{array}{l}33.36 \pm \\
2.62\end{array}$ & $P=0.006$ & $\begin{array}{l}32.09 \pm \\
2.42\end{array}$ & NS \\
\hline & Dmean & $4.46 \pm 0.68$ & $4.83 \pm 0.68$ & $P=0.002$ & $4.39 \pm 0.71$ & NS \\
\hline & V5 & $\begin{array}{l}21.67 \pm \\
3.09\end{array}$ & $\begin{array}{l}22.99 \pm \\
3.06\end{array}$ & $P=0.010$ & $\begin{array}{l}19.85 \pm \\
5.45\end{array}$ & $P=0.035$ \\
\hline & V10 & $\begin{array}{l}14.13 \pm \\
2.59\end{array}$ & $\begin{array}{l}15.36 \pm \\
2.69\end{array}$ & $P=0.004$ & $\begin{array}{l}13.88 \pm \\
2.68\end{array}$ & NS \\
\hline & V20 & $7.31 \pm 1.69$ & $7.93 \pm 1.71$ & $P=0.037$ & $7.14 \pm 1.63$ & NS \\
\hline & V30 & $2.86 \pm 0.87$ & $3.37 \pm 1.13$ & $P=0.012$ & $2.86 \pm 0.97$ & NS \\
\hline \multirow[t]{2}{*}{ Right Lung } & D02 & $4.48 \pm 3.12$ & $4.44 \pm 3.46$ & NS & $4.14 \pm 3.04$ & $P=0.030$ \\
\hline & Dmean & $0.80 \pm 0.54$ & $0.87 \pm 0.65$ & NS & $0.76 \pm 0.55$ & NS \\
\hline
\end{tabular}




\begin{tabular}{|c|c|c|c|c|c|c|}
\hline ROI & & $\mathrm{BH} 1$ & FTBH & $\begin{array}{l}\text { P-value } \\
\text { FTBH }\end{array}$ & RBH & $\begin{array}{l}\text { P-value } \\
\text { RBH }\end{array}$ \\
\hline & V5 & $2.73 \pm 4.44$ & $3.06 \pm 5.55$ & NS & $2.46 \pm 4.40$ & $P=0.014$ \\
\hline & V10 & $0.63 \pm 0.83$ & $0.61 \pm 1.08$ & NS & $0.49 \pm 0.81$ & NS \\
\hline & V20 & $0.06 \pm 0.18$ & $0.01 \pm 0.03$ & NS & $0.01 \pm 0.03$ & NS \\
\hline & V30 & $0.02 \pm 0.07$ & $0.00 \pm 0.00$ & NS & $0.00 \pm 0.00$ & NS \\
\hline \multirow[t]{6}{*}{ Thyroid } & D02 & $\begin{array}{l}18.91 \pm \\
11.21\end{array}$ & $\begin{array}{l}20.10 \pm \\
14.87\end{array}$ & NS & $\begin{array}{l}21.53 \pm \\
12.09\end{array}$ & $P=0.042$ \\
\hline & Dmean & $3.38 \pm 1.79$ & $4.24 \pm 3.25$ & NS & $3.76 \pm 2.08$ & NS \\
\hline & V5 & $\begin{array}{l}14.37 \pm \\
9.27\end{array}$ & $\begin{array}{l}17.28 \pm \\
15.80\end{array}$ & NS & $\begin{array}{l}16.18 \pm \\
10.92\end{array}$ & NS \\
\hline & V10 & $7.72 \pm 6.87$ & $\begin{array}{l}11.10 \pm \\
11.90\end{array}$ & NS & $9.69 \pm 7.92$ & NS \\
\hline & V20 & $3.39 \pm 4.48$ & $6.04 \pm 7.76$ & NS & $4.55 \pm 4.93$ & NS \\
\hline & V30 & $1.27 \pm 2.42$ & $2.88 \pm 4.69$ & NS & $1.79 \pm 2.61$ & $P=0.043$ \\
\hline \multirow[t]{6}{*}{ Esophagus } & D02 & $\begin{array}{l}15.97 \pm \\
4.64\end{array}$ & $\begin{array}{l}22.37 \pm \\
9.69\end{array}$ & $P=0.003$ & $\begin{array}{l}16.60 \pm \\
7.72\end{array}$ & NS \\
\hline & Dmean & $2.44 \pm 0.77$ & $3.41 \pm 1.51$ & $P=0.002$ & $2.71 \pm 1.57$ & NS \\
\hline & V5 & $\begin{array}{l}13.00 \pm \\
7.00\end{array}$ & $\begin{array}{l}16.14 \pm \\
7.98\end{array}$ & $P=0.007$ & $\begin{array}{l}14.00 \pm \\
10.97\end{array}$ & NS \\
\hline & V10 & $6.71 \pm 4.33$ & $\begin{array}{l}10.54 \pm \\
6.36\end{array}$ & $P=0.003$ & $7.82 \pm 7.49$ & NS \\
\hline & V20 & $1.10 \pm 0.97$ & $3.92 \pm 4.08$ & $P=0.004$ & $1.78 \pm 2.59$ & NS \\
\hline & V30 & $0.04 \pm 0.08$ & $1.36 \pm 2.28$ & $P=0.018$ & $0.34 \pm 1.11$ & NS \\
\hline \multirow{6}{*}{$\begin{array}{l}\text { Contralateral } \\
\text { breast }\end{array}$} & D02 & $5.28 \pm 2.32$ & $5.57 \pm 2.62$ & NS & $5.47 \pm 2.48$ & $P=0.046$ \\
\hline & Dmean & $1.11 \pm 0.45$ & $1.12 \pm 0.48$ & NS & $1.12 \pm 0.46$ & $P=0.048$ \\
\hline & V5 & $3.17 \pm 2.88$ & $3.23 \pm 3.21$ & NS & $3.23 \pm 2.86$ & NS \\
\hline & V10 & $\begin{array}{l}0.034 \pm \\
0.51\end{array}$ & $0.48 \pm 0.67$ & $P=0.024$ & $0.42 \pm 0.62$ & NS \\
\hline & V20 & $0.01 \pm 0.03$ & $0.02 \pm 0.08$ & NS & $0.01 \pm 0.04$ & NS \\
\hline & V30 & $0.00 \pm 0.00$ & $0.00 \pm 0.00$ & NS & $0.00 \pm 0.00$ & NS \\
\hline
\end{tabular}

\section{Discussion}


This research evaluates the clinical impact of positional changes of RBH or FTBH in WBI + RNI including the LNN MI in prone position. We demonstrated that there is more positional variation for nodal TVs, LAD, esophagus and thyroid in $\mathrm{RBH}$ than previously observed for the breast itself ${ }^{13}$. The positional changes vary markedly more for all ROls in FTBH. The impact of positional variation in $\mathrm{RBH}$ on dosimetry was limited, but the impact of positional variation in FTBH was more important.

Only few papers report on WBI + RNI in prone position, always with free breathing ${ }^{9-12,24,25}$ and most do not treat LNN MI.

In a former publication from our group, Speleers et al. ${ }^{12}$ report on WBI + RNI including LNN MI in supine and prone position comparing photons with protons, but with free breathing. Compared to their free breathing prone position, mean doses for heart (4.3 vs $2.47 \mathrm{~Gy}$ ), LAD (9.0 vs $5.99 \mathrm{~Gy}$ ), ipsilateral lung (5.3 vs $4.46 \mathrm{~Gy}$ ) and contralateral lung ( $0.91 \mathrm{vs} 0.8 \mathrm{~Gy}$ ) are in favour of breathhold. Our FTBH OAR doses are very similar to their free breathing treatment. However, proton treatment - either in supine or in prone position - is superior to our results for sparing OARs. Kainz et al. report WBI + RNI including LNN MI in prone position using helical tomotherapy, but using a different dose prescription of $45 \mathrm{~Gy}$ and a different prone positioning setup ${ }^{10}$. Their mean OAR doses for left sided breast cancer patients are all higher than the ones reported in our trial by a factor exceeding the ratio of prescribed doses (factor 1.12): heart (8.7 vs $2.47 \mathrm{~Gy}$ ), ipsilateral lung (10.0 vs $4.46 \mathrm{~Gy}$ ), contralateral lung (3.8 vs $0.8 \mathrm{~Gy}$ ), esophagus ( 8.7 vs 2.44 Gy), thyroid (22.6 vs $3.38 \mathrm{~Gy}$ ), contralateral breast (2.4 vs $1.11 \mathrm{~Gy}$ ). The difference can be explained by a difference in prone set-up and the non-coplanar treatment possibilities of the prone crawl breast couch ${ }^{16}$ used in our trial.

This trial did not intend to evaluate the differences between breathhold or free breathing on OARs, but rather the consistency of intra-fraction RBH and dosimetrical impact of FTBH. Nevertheless, doses to OARs in FTBH anatomy are similar to those in a dedicated FB plan in prone position and better than in supine position ${ }^{10,12}$. The difference in overlap measures between breathhold and free breathing anatomy confirms prior observations that the heart changes position away from the treated fields ${ }^{26}$ and that breathhold increases lung volume decreasing the percentage of lung tissue being exposed to the treatment beams. Given these data, this trial indicates a probable benefit of breathhold, and this is confirmed in data from our group (manuscript under review, preprint available) ${ }^{27}$.

Because treatment duration for each fraction is too long for each patient to undergo in a single breathhold without further training, our trial investigated whether the treatment is robust enough for RBH during a single treatment fraction. Our data demonstrate low variability in location and in dosimetric impact.

The RBH technique used in this trial has the benefit of being very easily applicable. It has proven to be reproducible in $\mathrm{WBI}$, with almost no movement of the treated breast ${ }^{13}$. We only use 2 Respisens magnetic sensors used to monitor if a breathhold is being performed and interrupt the beam manually if 
the patient fails to maintain breathhold. No automatic gating is required. This trial now shows that this technique can be extended to WBI + RNI including LNN MI, when a prone crawl breast couch is used.

Comparison with FTBH demonstrated the impact of patients not maintaining breathhold for the entire treatment, which is highly unlikely. As shown, dose distribution of the TVs is nearly identical, except for a slight underdosage to the LNN MI but without significant change in mean dose to this region. This could be of importance for the benefits that are observed in trials that include the LNN MI in the regional nodal targets ${ }^{28-31}$. These trials, however, used standard field setups for all patients. Borm et al. ${ }^{32}$ investigated the dose distributions that are achieved using these setups for some of the landmark trials in regional nodal irradiation. The mean dose to the LNN MI region in MA.20 and EORTC 10981-22023 was about 37.8 and $41.8 \mathrm{~Gy}$, respectively, which corresponds to $76 \%$ and $84 \%$ of the prescribed dose of $50 \mathrm{~Gy}$ to this region. In our trial, the mean dose to the LNN MI region was not significantly different between $\mathrm{RBH}$ and FTBH, and the average minimum dose in FTBH is still $85 \%$ of the prescribed dose. The same can be said for region LNN II, that received a minimum dose of $91 \%$ of the prescription dose in our trial, still higher than the mean dose reported in this region for the MA.20 group that had more than 10 nodes removed or had less than 3 affected nodes, namely $88 \%$ of the prescription dose.

Furthermore, our minimal dose to the CTV LNN, which we consider the unoperated part of the axilla during axillary lymph node dissection (axillary regions II-III-IV), is still covered with a minimal dose of $91 \%$ of the prescribed dose in the case of FTB. In the current era of CT dose calculation with patient level optimisation and intensity modulation, this is certainly a suboptimal result. But this result is still superior to the classic field setups on which our current evidence for regional nodal irradiation is based.

Unsurprisingly, we observed significantly higher OAR doses in FTBH, especially for heart, LAD, lungs and esophagus.

We did not include axillary region I into the TVs. This is the largest lymph node region, and including it might increase heart and lung doses, especially since this region is not covered "accidentally" in prone irradiation of the breast ${ }^{33}$. However, this region is surgically cleared in axillary lymph node dissection, whereas the other lymph node regions are (partly) avoided during standard axillary dissection. Therefore, our institution only includes level I in the case of positive sentinel node biopsy and no axillary dissection (AMAROS) ${ }^{34}$ or a high positive/total removed nodal ratio ${ }^{35}$.

Although the dose transposition method in itself proves that RBH can be used and FTBH leads to only small underdosages, we also report the overlap measures. Their importance is that they show that ROls can have very dissimilar position between $\mathrm{BH}$ and FTBH (essentially free breathing) anatomy. The latter anatomy is often the one that is used for online CBCT or EPID matching prior to delivery of a treatment fraction. Matching on a different anatomy than the one used in treatment requires that the change from free breathing to breathhold position is consistently the same. In this trial, we only validated $\mathrm{RBH}$, but not the validity of matching in free breathing and treating in breathhold. Therefore, we propose acquiring CBCT or EPID in breathhold and matching to the BH scan anatomy. 
A point for improvement in this trial is that we delineated all regions of interest on CT images acquired without contrast while the RBH scan was a low dose $\mathrm{CT}$, which is inherently more prone to artefacts. But because the RBH scan had no therapeutic implications, it was deemed more ethical to perform a low dose CT. Despite these issues, we found the delineation was not hampered for delineation of the TVs and major OARs, only the thyroid and esophagus were more difficult to identify on the RBH scan.

One caveat is that we only evaluated intra-fraction reproducibility of $\mathrm{RBH}$, and not inter-fraction reproducibility. However, given the treatment robustness that we showed in this manuscript, it stands to reason that inter-fraction variability will have less impact than complete FTBH. Therefore inter-fraction variability will probably result in doses ranging between what we report for FTBH and RBH.

Our results shows that $\mathrm{RBH}$ can be performed using a simple technique and without fear for clinically relevant differences between the intended and delivered plan, provided that there is adequate image guided patient position verification at the start of each session. In the event of FTBH, TV coverage in photon radiotherapy will still be adequate, whereas OAR dose will increase.

\section{Conclusion}

When using RBH, OAR and TV position in prone crawl position is reproducible for all the large ROls while smaller volumes of interest, such as the LAD and the IMN show poorer overlap. All ROIs have similar dosimetry in RBH. For FTBH, TVs remain adequately covered but overlap is poor for most ROls, and the heart, LAD, ipsilateral lung and esophagus receive higher doses. RBH shows the robustness required for clinical use, but CBCT should preferentially also be performed in $\mathrm{BH}$.

\section{Declarations}

\section{Data Availability}

Data analysed during this study are available from the corresponding author on reasonable request within the confines of EU General Data Protection Regulations.

\section{Acknowledgements}

This research was supported by Cancer Plan Action 29 by the Federal Public Service of Health, Food Chain Safety and Environment, Belgium; by Foundation against Cancer grants (FAF-C/2016/854 and FAFC/2018/1190) and by a Career Catalyst Grant from Susan G. Komen® (Grant 377841). Prototype research was funded by StarTT 241 grant of the Industrial Research Fund, Ghent University. Liv Veldeman holds a Clinical Mandate of Foundation against Cancer.

\section{Author contributions}

PD wrote the main manuscript and participated in designing the trial together with HD, WDN and LV. HVH, AVG and GP performed the patient positioning and CT simulations. Structure delineation was done by 
$\mathrm{CM}, \mathrm{AVG}$ and GP for OARs and revised by PD. Target volumes were delineated by VV and PD, with PD having the deciding vote in case of disagreement. BS designed the treatment plans for this trial. LP and WDG were involved in calculating overlap measures and transposing the treatment beams and extracting dose parameters. Data analysis was performed by PD. Graphs were designed by LV, MS and PD, and were made by PD using the ggplot2 package. All authors contributed to the manuscript and agree with its current form.

\section{Competing interests statement}

Ghent University owns the patent application entitled Radiotherapy Board and Couch [W02015144654A1] filed on 25.03.2014 for which Wilfried De Neve, Bruno Speleers, Bert Boute and Liv Veldeman own the intellectual rights.

\section{References}

1. Darby, S. C. et al. Risk of ischemic heart disease in women after radiotherapy for breast cancer. $N$ Engl J Med. 368, 987-998 https://doi.org/10.1056/NEJMoa1209825 (2013).

2. Bartlett, F. R. et al. The UK HeartSpare Study (Stage II): Multicentre Evaluation of a Voluntary Breathhold Technique in Patients Receiving Breast Radiotherapy. Clin Oncol (R Coll Radiol). 29, e51-e56 https://doi.org/10.1016/j.clon.2016.11.005 (2017).

3. Mulliez, T. et al. Heart dose reduction by prone deep inspiration breath hold in left-sided breast irradiation. Radiother Oncol. 114, 79-84 https://doi.org/10.1016/j.radonc.2014.11.038 (2015).

4. Formenti, S. C., DeWyngaert, J. K., Jozsef, G. \& Goldberg, J. D. Prone vs supine positioning for breast cancer radiotherapy. JAMA. 308, 861-863 https://doi.org/10.1001/2012.jama.10759 (2012).

5. Lymberis, S. C. et al. Prospective assessment of optimal individual position (prone versus supine) for breast radiotherapy: volumetric and dosimetric correlations in 100 patients. Int J Radiat Oncol Biol Phys. 84, 902-909 https://doi.org/10.1016/j.ijrobp.2012.01.040 (2012).

6. Pandeli, C., Smyth, L. M. L., David, S. \& See, A. W. Dose reduction to organs at risk with deepinspiration breath-hold during right breast radiotherapy: a treatment planning study. Radiation oncology (London, England). 14, 223 https://doi.org/10.1186/s13014-019-1430-x (2019).

7. Yeung, R. et al. Cardiac dose reduction with deep inspiration breath hold for left-sided breast cancer radiotherapy patients with and without regional nodal irradiation. Radiation oncology (London, England). 10, 200 https://doi.org/10.1186/s13014-015-0511-8 (2015).

8. Shin, S. M. et al. Breast, chest wall, and nodal irradiation with prone set-up: Results of a hypofractionated trial with a median follow-up of 35 months. Pract Radiat Oncol. 6, e81-88 https://doi.org/10.1016/j.prro.2015.10.022 (2016).

9. Deseyne, P. et al. Whole breast and regional nodal irradiation in prone versus supine position in left sided breast cancer. Radiat Oncol. 12, 89 https://doi.org/10.1186/s13014-017-0828-6 (2017). 
10. Kainz, K. et al. Simultaneous irradiation of the breast and regional lymph nodes in prone position using helical tomotherapy. Br J Radiol. 85, e899-905 https://doi.org/10.1259/bjr/18685881 (2012).

11. Gielda, B. T., Strauss, J. B., Marsh, J. C., Turian, J. V. \& Griem, K. L. A Dosimetric Comparison Between the Supine and Prone Positions for Three-Field Intact Breast Radiotherapy. Am J Clin Oncol-Canc. 34, 223-230 https://doi.org/10.1097/COC.0b013e3181dbb9c1 (2011).

12. Speleers, B. A. et al. Comparison of supine or prone crawl photon or proton breast and regional lymph node radiation therapy including the internal mammary chain. Scientific reports. 9, 4755 https://doi.org/10.1038/s41598-019-41283-1 (2019).

13. Mulliez, T. et al. Reproducibility of deep inspiration breath hold for prone left-sided whole breast irradiation. Radiat Oncol. 10, 9 https://doi.org/10.1186/s13014-014-0313-4 (2015).

14. Borm, K. J., Oechsner, M., Combs, S. E. \& Duma, M. N. Deep-Inspiration Breath-Hold Radiation Therapy in Breast Cancer: A Word of Caution on the Dose to the Axillary Lymph Node Levels. International journal of radiation oncology, biology, physics. 100, 263-269 https://doi.org/10.1016/j.jijobp.2017.09.026 (2018).

15. Pazos, M. et al. Dose variability in different lymph node levels during locoregional breast cancer irradiation: the impact of deep-inspiration breath hold. Strahlentherapie und Onkologie: Organ der Deutschen Rontgengesel/schaft ... et al]. 195, 13-20 https://doi.org/10.1007/s00066-018-1350-y (2019).

16. Boute, B. et al. Potential benefits of crawl position for prone radiation therapy in breast cancer. $J$ Appl Clin Med Phys. 18, 200-205 https://doi.org/10.1002/acm2.12118 (2017).

17. Remouchamps, V. M. et al. The use of magnetic sensors to monitor moderate deep inspiration breath hold during breast irradiation with dynamic MLC compensators. Radiother Oncol. 82, 341-348 https://doi.org/10.1016/j.radonc.2006.11.015 (2007).

18. Offersen, B. V. et al. ESTRO consensus guideline on target volume delineation for elective radiation therapy of early stage breast cancer. Radiother Oncol. 114, 3-10 https://doi.org/10.1016/j.radonc.2014.11.030 (2015).

19. Offersen, B. V. et al. ESTRO consensus guideline on target volume delineation for elective radiation therapy of early stage breast cancer, version 1.1. Radiother Oncol. 118, 205-208 https://doi.org/10.1016/j.radonc.2015.12.027 (2016).

20. Verhoeven, K. et al. Vessel based delineation guidelines for the elective lymph node regions in breast cancer radiation therapy - PROCAB guidelines. Radiother Oncol. 114, 11-16 https://doi.org/10.1016/j.radonc.2014.11.008 (2015).

21. Verhoeven, K. et al. Vessel based delineation guidelines for the elective lymph node regions in breast cancer radiation therapy - PROCAB guidelines. Radiother Oncol. 118, 209-210 https://doi.org/10.1016/j.radonc.2015.12.005 (2016).

22. Feng, M. et al. Development and validation of a heart atlas to study cardiac exposure to radiation following treatment for breast cancer. Int J Radiat Oncol Biol Phys. 79, 10-18 https://doi.org/10.1016/j.jirobp.2009.10.058 (2011). 
23. Zou, K. H. et al. Statistical validation of image segmentation quality based on a spatial overlap index. Acad Radiol. 11, 178-189 (2004).

24. Coffey, A., Renucci, L., Hunzeker, A. \& Lenards, N. Whole breast nodal irradiation using supine VMAT and prone 3D planning: A case study. Med Dosim. 42, 185-189 https://doi.org/10.1016/j.meddos.2017.02.007 (2017).

25. Mason, N., Macfarlane, D., Guidi, R., Owen, R. \& Poulsen, M. A prone technique for treatment of the breast, supraclavicular and axillary nodes. J Med Imaging Radiat Oncol. 56, 362-367 https://doi.org/10.1111/j.1754-9485.2012.02389.x (2012).

26. Mulliez, T. et al. Deep inspiration breath hold in the prone position retracts the heart from the breast and internal mammary lymph node region. Radiother Oncol. 117, 473-476 https://doi.org/10.1016/j.radonc.2015.09.030 (2015).

27. Speleers, B. et al. Comparison between deep inspiration breath hold and shallow breathing for prone photon or proton irradiation of breast and regional lymph nodes. Preprint athttps://www.researchsquare.com/article/rs-115182/v1(2020).

28. Poortmans, P. M. et al. Internal mammary and medial supraclavicular lymph node chain irradiation in stage I-III breast cancer (EORTC 22922/10925): 15-year results of a randomised, phase 3 trial. Lancet Oncol. https://doi.org/10.1016/S1470-2045(20)30472-1 (2020).

29. Thorsen, L. B. et al. DBCG-IMN: A Population-Based Cohort Study on the Effect of Internal Mammary Node Irradiation in Early Node-Positive Breast Cancer. J Clin Oncol. 34, 314-320 https://doi.org/10.1200/JC0.2015.63.6456 (2016).

30. Whelan, T. J. et al. Regional Nodal Irradiation in Early-Stage Breast Cancer. N Engl J Med. 373, 307316 https://doi.org/10.1056/NEJMoa1415340 (2015).

31. Budach, W. et al. Adjuvant radiation therapy of regional lymph nodes in breast cancer - a metaanalysis of randomized trials- an update. Radiat Oncol. 10, 258 https://doi.org/10.1186/s13014-0150568-4 (2015).

32. Borm, K. J. et al. Irradiation of regional lymph node areas in breast cancer - Dose evaluation according to the Z0011, AMAROS, EORTC 10981-22023 and field design. Radiotherapy and oncology: journal of the European Society for Therapeutic Radiology and Oncology 142, 195-201, doi:10.1016/j.radonc.2019.08.021 (2020).

33. Alonso-Basanta, M., Ko, J., Babcock, M., Dewyngaert, J. K. \& Formenti, S. C. Coverage of axillary lymph nodes in supine vs. prone breast radiotherapy. Int J Radiat Oncol Biol Phys. 73, 745-751 https://doi.org/10.1016/j.jijobp.2008.04.040 (2009).

34. Donker, M. et al. Radiotherapy or surgery of the axilla after a positive sentinel node in breast cancer (EORTC 10981-22023 AMAROS): a randomised, multicentre, open-label, phase 3 non-inferiority trial. Lancet Oncol. 15, 1303-1310 https://doi.org/10.1016/S1470-2045(14)70460-7 (2014).

35. Fortin, A., Dagnault, A., Blondeau, L., Vu, T. T. \& Larochelle, M. The impact of the number of excised axillary nodes and of the percentage of involved nodes on regional nodal failure in patients treated 
by breast-conserving surgery with or without regional irradiation. International journal of radiation oncology, biology, physics. 65, 33-39 https://doi.org/10.1016/j.jirobp.2005.12.014 (2006).

\section{Figures}
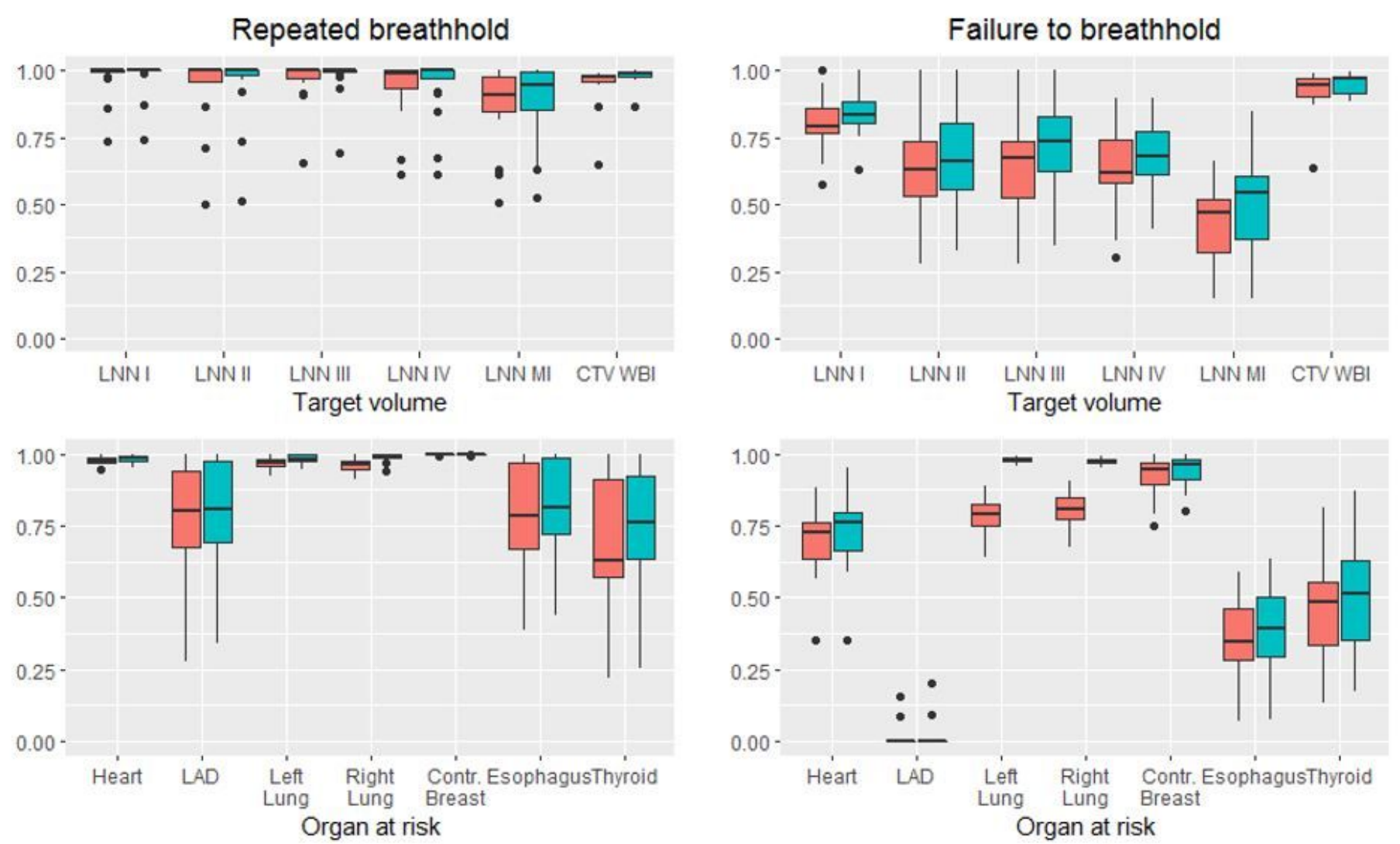

Index Type Dice Similarity Coefficient

\section{Figure 1}

Boxplot of the overlap measurements for targets and organs at risk for the repeated breathhold and failure to breathhold setting. The top row shows target volumes and the bottom row shows OARs, while the first column shows the situation for repeated breathhold and the second column shows the situation for failure to breathhold. Whiskers show 1.5 * interquartile range, outliers plotted as dots. Overlap index is consistently higher than Dice similarity coefficient for the same ROls in the same breathing phase setting. Small volumes have poorer overlap measurements. This is especially visible in failure to breathhold setting. 

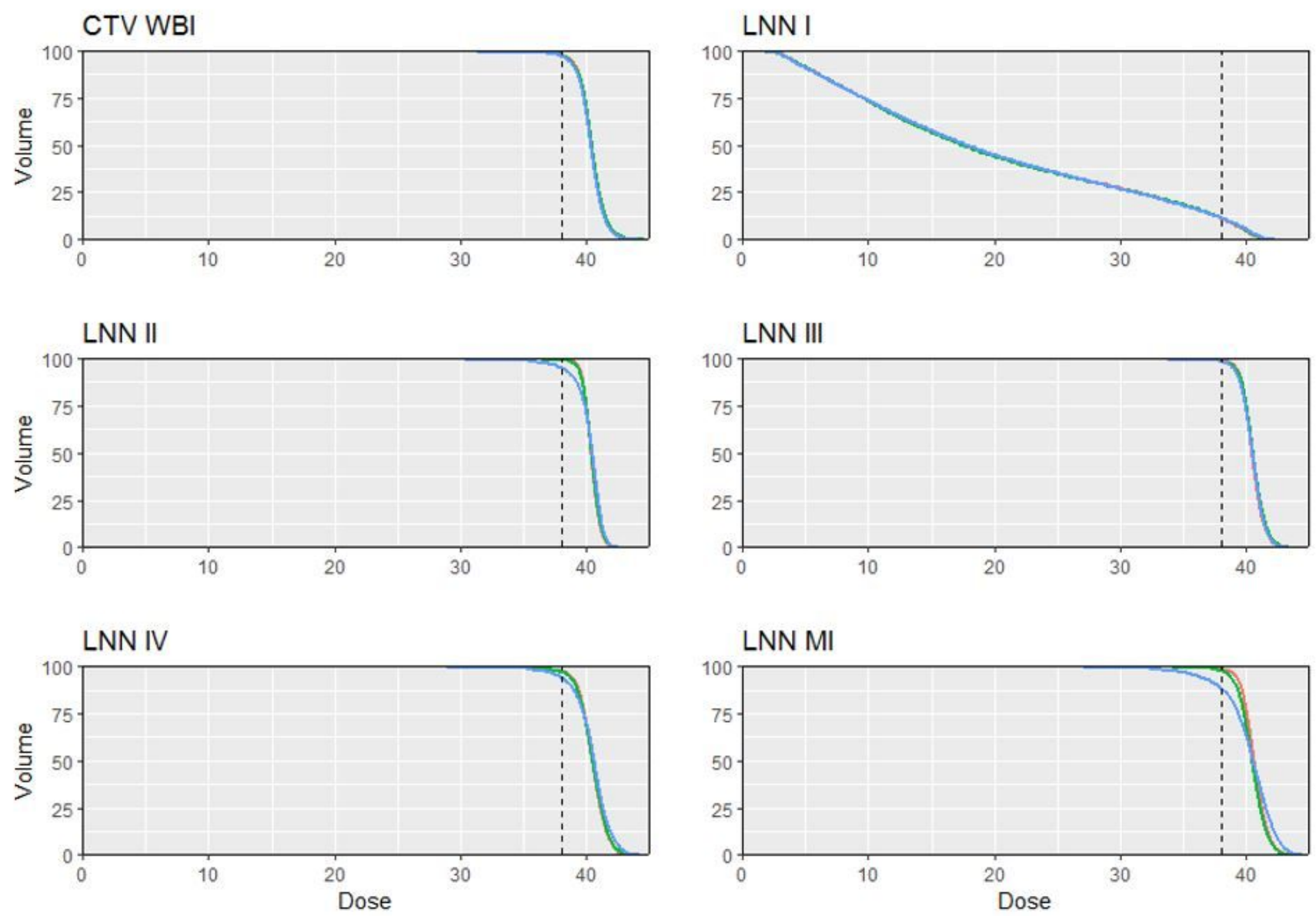

Breathing anatomy - Breathhold — Repeated breathhold - Failure to breathhold

\section{Figure 2}

Dose-volume histogram showing mean (of patients) minimum dose received by given percentage of target volume. Almost all lines overlap, indicating the robustness of a photon radiotherapy treatment: only the internal mammary nodes (LNN MI) experienced higher dose inhomogeneity and underdosage in failure to breathhold anatomy. The vertical interrupted line represents $95 \%$ of the prescription dose. 

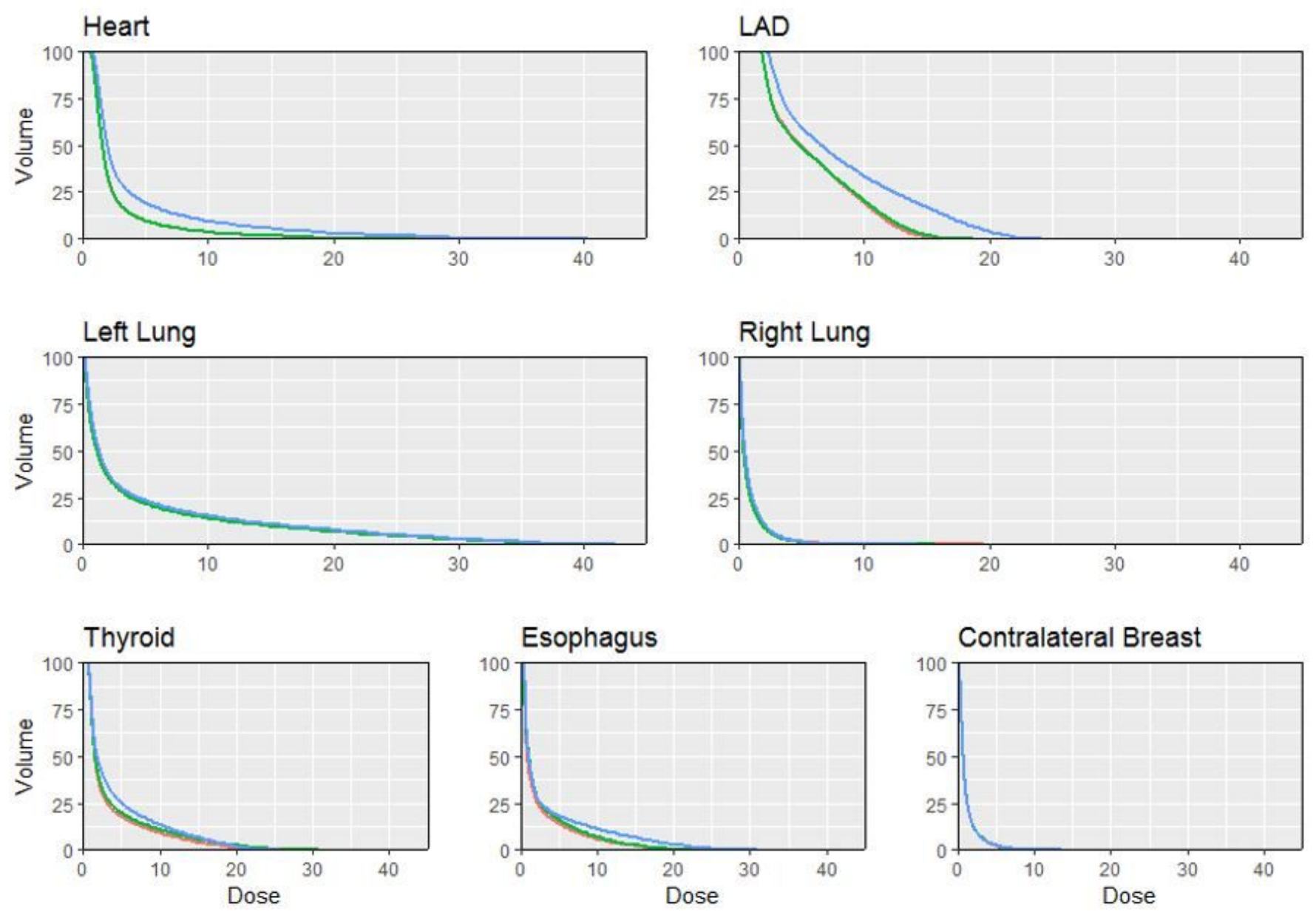

Breathing anatomy - Breathhold - Repeated breathhold - Failure to breathhold

\section{Figure 3}

Dose-volume histogram showing mean (of patients) minimum dose received by given percentage of target volume. Contrary to TVs, failure to breathhold causes heart and LAD doses to increase, as compared to breathhold anatomy. Lines for repeated breathhold overlap, showing that although statistical differences exist, these are likely not relevant. 